\title{
Some referents and measures of nonverbal behavior'
}

\section{ALBERT MEHRABIAN, UNIVERSITY OF CALIFORNIA, LOS ANGELES, California 90024}

This paper summarizes some of the measures of nonverbal behavior that have been found to be significant indicators of a communicator's attitude toward, status relative to, and responsiveness to his addressee. The nonverbal cues considered include posture, position, movement, facial, and implicit verbal cues. In addition to providing criteria for the scoring of these cues, experimental findings that relate to the various cues are summarized.

This paper summarizes criteria for the scoring of nonverbal behaviors and suggests groupings on the basis of findings from attitude and status communication. In attempting to categorize and score nonverbal behaviors, there are of course several theoretical and methodological alternatives. Our selection of categories used the following conceptualization as a guideline. If nonverbal behaviors are part of communication, then what are the major referents of these behaviors? Earlier work in this area sought to identify isolated moods and feelings (e.g., fear, anger, happiness) as referents of particular facial, movement, postural, and vocal cues. Such research led to the identification of seven feelings that were coded into, and decoded from, behaviors with considerable consensus (e.g., Tomkins \& McCarter, 1964; Woodworth \& Schlosberg, 1954, Ch. 5). These results led to the further development of measures of individual differences in the accurate communication of moods or feelings (e.g., Davitz, 1964).

An alternate attempt conceptualized the referents of nonverbal communication in terms of a multidimensional framework. Schlosberg (1954) suggested that facial movements, for instance, could be characterized within the three-dimensional framework of pleasantness-unpleasantness, sleep-tension, and attentionrejection. Thus anger would be characterized with a slight degree of rejection and high degrees of tension and unpleasantness. This scheme, then, made possible a parsimonious characterization of the major referents of nonverbal behavior.

More recent factor-analytic work provided additional support for the use of a limited set of dimensions to characterize the referents of nonverbal communication. Williams and Sundene (1965) and Osgood (1966) conducted studies whose results paralleled quite closely those obtained from more general studies of communication by Osgood, Suci, and Tannenbaum (1957). The findings indicated that, at least for facial, vocal, and/or combined facial-vocal communications, the major referential factors were: general evaluation, social control, and activity. These three dimensions were thus seen to constitute not only the referents of verbal concepts, as found by Osgood, Suci, and Tannenbaum, but also the basic referents of nonverbal behavior. Such a corroboration of results, despite the heterogeneity of communication cues, is especially reassuring, and led to the present groupings of scoring criteria.

More specifically, the measures summarized below were developed with the intent of assessing evaluative attitudes (i.e., liking, preference, and degree of positive conceptual evaluation), social control (i.e., communicated status or potency), and responsiveness (i.e., activity level) of a communicator toward his addressee.

\section{Posture and Position}

With the preceding general outline of nonverbal communication referents, it is now possible to proceed to a summary statement of the nonverbal behaviors that have been found relevant for the communication of these referents.

According to the criteria of Table 1, there are two sets of behaviors among posture and position cues for communicating attitude and status. When available, reliability scores are reported within paren theses for the various cues of Table 1 .

The summary terms, immediacy or proxemics (e.g., Hall, 1963, 1966; Mehrabian, 1967), are used to refer to one set of measures that have been found to relate primarily to the attitude of a communicator toward his addressee. These are: touching, distance, forward lean, eye contact, and body orientation. Increasing degrees of immediacy correspond to greater degrees of touching, forward lean, eye contact, and directness of body orientation, and smaller distances. Although greater degrees of immediacy primarily communicate more liking of a communicator toward his addressee, this relation is reversed when the communicator is tense. Thus, immediacy is directly correlated with positive attitude for moderately and very relaxed postures, but is negatively correlated with positive attitude for the less frequent tense postures (Mehrabian, 1969).

The five immediacy cues of touching, distance, forward lean, eye contact, and orientation are grouped and defined on the basis of experimental findings as well as conceptual considerations. Distance and forward lean were consistently found to be associated with more positive attitude. This similarity in the significance of the two cues is partially understood by noting that when due to the fixed position of furniture, distance variations are not available for the communication of attitude, a communicator can still decrease or increase his distance from the addressee by assuming either a reclining position or a forward-leaning position. Even when a communicator has a choice of furniture at various distances to an addressee, he can still use forward lean as an additional cue for communicating greater degrees of closeness to the addressee. Thus, touching, distance, and forward lean are readily related as variations in the degree of physical proximity of a communicator with an addressee.

Immediacy is defined in a somewhat general form as the extent to which communication behaviors enhance closeness to and nonverbal interaction with another. Thus, for all the five cues, greater immediacy is due to increasing degrees of physical proximity and/or increasing perceptual availability of the communicator to the addressee.

Although there are no experimental data available for touching in relation to attitude, the preceding conceptualization plus informal observations led us to include touching as an important variable of immediacy. Of the five variables, body orientation has yielded the weakest relationships with attitude, and therefore is considered the least important immediacy cue. However, in the one experiment (Mehrabian, 1967) in which the effects of body orientation and eye contact of female communicators were experimentally separated, more direct body orientation was associated with a more positive attitude.

In considering the preceding grouping of nonverbal immediacy cues, it is of some interest to note that variations in the immediacy of verbal cues have also been identified and investigated. Studies have consistently provided support for the 


\section{IMMEDIACY CUES-Criteria listed in order of importance.}

A. Touching: there is bodily contact between the communicator and the addressee, as would be the case in holding hands, or shoulders touching. In the case of this and the eye contact measure noted below, the duration of touching (or of eye contact), expressed as a fraction of the total time when the communicator and addressee are in each other's presence, is the appropriate measure. For all of the remaining measures average values over time are used (e.g., average distance during a 5 -min conversation between the communicator and addressee).

B. Distance (0.95): physical distance separating the communicator from the addressee. In two studies involving seated communicators (Mehrabian, 1968b; Mehrabian \& Friar, in press) distance was specifically scored as follows.

1. Straight-ahead distance: the distance from the front of the addressee's chair to the center spoke of the communicator's chair. This is measured by the $9 \times 9$ in. tiles on the floor, with the tile immediately below the front of the addressee's chair being zero-the distance is measured to the nearest one-half tile.

2. Lateral distance: the distance the communicator is to the right or left of an imaginary plane that would cut the addressee's chair bilaterally in half. This distance is measured by the tiles on the floor from the imaginary plane to the center spoke of the communicator's chair-the distance is measured to the nearest one-half tile.

In one study involving standing communicators (Mehrabian, 1968a), the following criteria were used for distance.

1. Straight-ahead distance: measured from front line of addressee in terms of number of tiles to nearest half, up to a line that is perpendicular to the straight ahead and that passes near the center portion of the foot on which the communicator is resting. If he is resting on both feet, then a point in between the two feet is used.

2. Lateral distance: measured similarly to the straight-ahead distance.

Total distance is, of course, the relevant measure and is based on the preceding straight-ahead and lateral distance scores.

C. Forward Lean (0.87): the number of degrees that a plane defined by a line from the communicator's shoulders to his hips is away from the vertical plane. Angles are measured in units of $10 \mathrm{deg}$ and whereas reclining angles are scored as negative, forward-leaning angles are scored as positive.

D. Eye Contact (0.55): fraction of the duration of interaction (i.e., when the communicator and the addressee are in each other's presence) when the communicants look into each other's eyes.

E. Orientation (0.90): the number of degrees a plane perpendicular to the plane of the communicator's shoulders is turned away from the median plane of the addressee. This angle is estimated to the nearest 10 deg and cannot exceed $180 \mathrm{deg}$.

NOTE: Increasing degrees of touching, forward lean, and eye contact, whereas decreasing degrees of distance and orientation are more immediate. With the exception of the forward-lean angle, which is not scored for standing positions, all the criteria above are scored for both seated and standing positions.

\section{RELAXATION CUES-Criteria listed in approximate order of importance.}

\section{A. Arm Position Asymmetry (0.87).}

0 : Symmetrical position of the arms: for example, hands clasped at the midsection, arms folded symmetrically, or both hanging straight down or akimbo while standing.

1: Slight asymmetry in the position of the arms: for example, both hands resting on the lap of the communicator, but one is from 2 to 5 in. more forward than the other, or one hand clasps the other at the wrist.

2: Moderate asymmetry in the position of the arms: for example, one hand holds an elbow or the upper arm whereas the other hand is free, one arm hanging loosely and the other hanging by a finger which is stuck in a pocket.

3: Extreme asymmetry in the position of the arms: for example, one arm in lap and the other hooked over the back of the chair, one hand stuck in a pocket and the other resting on knee or hanging loosely, or only one arm akimbo.

B. Sideways Lean (0.63): the number of degrees that a plane cutting the communicator's torso bilaterally in half is away from a plane cutting his chair bilaterally in half. This angle is estimated to the nearest $10 \mathrm{deg}$ and cannot exceed $90 \mathrm{deg}$.

\section{Leg Position Asymmetry (0.96)}

0 : Symmetrical position of the legs with both feet flat on the floor and the insteps touching.

1: Symmetrical position of the legs with both feet flat on the floor and the insteps not touching.

2: Asymmetrical stance of the legs with both feet resting flat on the floor, such as when one foot is moved to a more forward position.

3: Asymmetrical stance of the legs with one or both feet partially lifted off the floor, as when there is a bend at the ankle and only an edge of the foot is resting on the floor, or when the legs are crossed while seated.

D. Hand Relaxation $(0.66)$

0 : Very tense; hands or fists are tightly clenched, or hands are clasping anything tightly, or hands are in motion, such as drumming fingers.

1: Moderately tense; loosely clasped or in loose fists or clasping any object or part of the body loosely.

2: Relaxed; fingers are extended but not stiffly.

E. Neck Relaxation (0.70)

0 : The head is not supported and the line of vision is pointing $10 \mathrm{deg}$ or more above the horizontal.

1: The head is not supported and the line of vision is within $10 \mathrm{deg}$ of the horizontal.

2: The head is supported as when resting on the back of a couch or is hanging so that the line of vision forms $\mathbf{1 0}$ or more degrees below the horizontal.

F. Reclining Angle (0.87): the number of degrees that a plane defined by a line from the communicator's shoulders to his hips is away from the vertical plane. Angles are measured in units of $10 \mathrm{deg}$ and whereas reclining angles are scored as positive, forward learning angles are scored as negative.

NOTE: Increasing degrees of each of the preceding criteria indicate more relaxation. With the exception of the sideways lean and reclining angles which are not scored for standing positions, all the criteria above are scored for both seated and standing positions. 
hypothesis that total immediacy in a verbalization is a direct correlate of positive attitude toward the contents of that verbalization. The conceptual basis, scoring criteria, and experimental studies of verbäl immediacy have been summarized by Wiener and Mehrabian (1968).

Postural relaxation is defined and measured in terms of the second set of nonverbal behaviors in Table $\mathrm{I}$ : arm asymmetry, sideways lean, leg asymmetry, hand relaxation, neck relaxation, and reclining angle, which is the converse of forward lean. Findings have indicated that leg asymmetry is an important determiner of relaxation. Further, Mehrabian and Williams (in press), who investigated the variables of arm openness, arm asymmetry, leg asymmetry, sideways lean, and reclining angle, together with a dozen nonverbal variables, obtained the following results. In two experiments a relaxation factor was defined by arm asymmetry, sideways lean, arm openness, leg asymmetry, and in one experiment only, reclining angle. Since arm asymmetry had the highest loading on the relaxation factor in both experiments, it can be used as an adequate measure of arm relaxation.

Results from this latter experiment and one by Mehrabian and Friar (in press) suggest the following ordering of the relaxation cues: arm asymmetry, sideways lean, leg asymmetry, hand relaxation, neck relaxation, and reclining angle. On both empirical as well as conceptual grounds, we feel reasonably confident in grouping the preceding cues as measures of communicator relaxation.

In contrast to the immediacy cues, the relaxation cues relate primarily to status variables. Thus leg asymmetry, neck, and hand relaxation were found to relate only to status differences; sideways lean exhibited a strong relationship to status differences, but only a weak effect in relation to attitudes. The findings relating the various relaxation cues to status consistently indicated that the greater a communicator's status relative to his addressee, the greater his relaxation. Further, findings associated with sideways lean, arm openness, and reclining angle suggested that attitude is a curvilinear function of relaxation. In the typical moderate to very relaxed range, attitude is negatively correlated with relaxation; in the atypical tense to moderately relaxed range, attitude is positively correlated with relaxation. Finally, although higher levels of immediacy do in most cases communicate more positive attitudes, there are some special cases in which relatively negative attitudes might be inferred, such as a tense or an unfamiliar communicator who is highly immediate (e.g., Mehrabian, 1969).

In the absence of additional experiments, one way to employ the list of relaxation cues in Table 1 is to transform the various relaxation indexes to standardized scores, and to simply sum the relaxation scores assigned to each of the body parts. This, of course, would imply that each relaxation cue is being assigned the same level of importance in determining total level of relaxation, and is a reasonably satisfactory tentative approach.

The contribution of the various immediacy cues to total immediacy is determined from findings relating these cues to attitudes. The experimental findings did suggest an ordering of the immediacy cues so that distance, forward lean, eye contact, and orientation would be assigned decreasing weights in determining total immediacy. For example, for seated postures, the findings of Mehrabian and Friar (in press) suggested the coefficients $-.57, .33$, and .29 as the contributions of distance, forward lean, and eye contact to total immediacy, provided of course that any measures employed were first standardized. In addition to these weights, the findings of Mehrabian (1967) suggest that the weight assigned to body orientation relative to eye contact is about $1 / 3$, at least for female communicators. Thus the approximate relative weights that can be assigned to the four immediacy cues that have been experimentally explored are as follows: -.6 for distance, .3 each for forward lean and eye contact, and -.1 for orientation. The signs indicate that larger distances and larger orientations are less immediate, and therefore are assigned negative values. Touching would seem to require the largest weight toward immediacy, although this remains to be shown.

In general, the effects of immediacy and relaxation on inferred attitude need to be specified separately for different levels of familiarity and different levels of actual status of a communicator relative to that of the addressee. Thus, for instance, variations in relaxation are not expected to have the same effects when a communicator and addressee are of the same status compared to when the communicator is of lower status than the addressee. Similarly, these effects are not expected to be the same when a communicator and addressee are familiar with each other in contrast to being unfamiliar.

\section{Movements, Facial Expressions, and Verbalizations}

Criteria for scoring certain categories of movement, facial expression, and verbalization, together with reliability figures, are given in Table 2. Although category systems for classifying movements have been proposed (e.g., Birdwhistell, 1952; Ekman \& Friesen, 1967), the findings relating to movements as well as verbal cues are few in number. The particular categories reported in Table 2 were selected because there were findings relating them to attitude and status. For instance, Rosenfeld (1966a) instructed some Ss to seek approval from their addressees and others to avoid approval. The behaviors of the Ss were rated on a series of nonverbal measures. Addressees next indicated their degree of approval for the communicator-Ss.

The following cues listed in terms of importance (i.e., $F$ values reported by Rosenfeld) served to differentiate the nonverbal behaviors of the approval-seeking from the approval-avoiding Ss: higher speech rate, lengthier verbal communications, more gesticulation, fewer negative head nods, more speech disturbances, more reinforcers to the addressee, more smiles, fewer self-references, and more positive head nods. (Incidentally, this and the following two lists do not include all the verbal cues investigated by Rosenfeld.)

To obtain a similar ranking of the nonverbal cues on the basis of positive impressions received by the addressees, we relied on average values from four specific correlations relating each of the nonverbal cues to approval received from naive listeners. These composite scores that were derived from Rosenfeld's data indicated that the following cues were correlated with positive impressions: higher rates of speech, positive head nods, and verbal reinforcers; infrequent self-manipulations and self-references; lengthier communications, more frequent smiles, speech disturbances, and gesticulations-listed in order from the more to the less important cues. Finally, the rank of importance of the various cues in communicating positive attitudes, as well as in being decoded as communicating positive attitudes, were summed to obtain the following final ranking of the cues that significantly determined more positive attitude communication: higher speech rate, lengthier communications in terms of total word output, more frequent verbal reinforcers emitted by the communicator, higher rates of positive head-nodding and gesticulation, fewer self-references, and higher rates of speech disturbance.

Several of the indexes noted in the preceding list were found in earlier experiments to be correlated with more positive attitudes. Verbal reinforcers, by definition, communicate a more positive attitude (e.g., Krasner, 1958) as do positive head nods (e.g., Matarazzo, Wiens, \& Saslow, 1965). Mehrabian (1965) found support for the hypothesis that lengthier communications are associated with more positive attitudes. There is only one item in 


\section{MOVEMENTS}

1. Trunk Swivel Movements (0.89): number of times $S$ swivels his body on a swivel chair. Score all movements which involve a rotation greater than $10 \mathrm{deg}$. If motions are cyclical rather than unidirectional, one complete cycle is scored as " 1 ."

2. Rocking Movements (0.91): number of times $S$ changes his angle of forward-back lean of torso by $10 \mathrm{deg}$ or more. Again cyclical movements (e.g., a forward-back rock) are scored as "1."

3. Head-Nodding Movements (0.80): number of cyclical up and down movements of the head each of which is scored as "1."

4. Gesticulation (0.98): number of movements of hands or of fingers, excluding the self-manipulatory movements scored below. This includes side-to-side, forward-back, and up-and-down movements. Cyclical movements (e.g., raising and lowering a finger) are scored as "1."

5. Self-manipulation (0.93): "Motion of a part of the body in contact with another-either directly or mediated by an instrument. Examples are scratching, rubbing, or tapping an arm or leg with finger or pen" (Rosenfeld, 1966b, p. 67), which are scored as in (4) above. In other words, single brief movements in one direction are scored once, and cyclical movements are scored once each. A brief (i.e., less than $5 \mathrm{sec}$ ) scratching movement is scored once only. Continuous movements are scored once every $5 \mathrm{sec}$.

6. Leg Movement (0.97): number of movements of the leg at the knee joint (e.g., placing one foot onto the other, crossing legs, or, if $S$ is short, letting feet hang and swing back and forth). Again any cyclical movements are scored as "1." Do not include in this measure any movements that are incidental to trunk swivel or rocking movements.

7. Foot Movement (0.87): number of movements of feet or number of times ankle is twisted (rotated). Again cyclical movements (e.g., turning the foot from side to side) are scored as " 1 ." Do not score movements incidental to leg movements.

\section{FACIAL EXPRESSIONS}

1. Facial Pleasantness (0.79): number of such positive expressions as smiles minus number of such negative expressions as frowns or sncers. Continuous expressions of either a positive or negative quality are scored once every 5 sec.

the above list, speech disturbances, that is inconsistent with earlier findings in terms of the interpretation assigned to it. Mahl (1959) or Kasl and Mahl (1965) provided evidence that speech disturbance frequency was a correlate of a communicator's anxiety level or discomfort. The weight of available empirical evidence does provide strong support for Mahl's hypothesis (e.g., Mahl \& Schulze, 1964). Thus, speech disturbance frequency should be correlated with negative attitude of a communicator toward ejther the addressee or the communication situation or both.

Among the indexes listed in Table 2, then, higher speech rate, longer communications, higher rates of gesticulation, lower rates of speech disturbance (or less halting quality of speech), more pleasant facial expressions, and frequent positive head nods communicate a more positive attitude. Further, the study by Mehrabian and Williams (in press) indicated that higher rates of rocking and gesticulation and lower rates of trunk swivel connote greater relaxation. In the same study, more speech volume, longer communications, higher speech rate, higher rates of leg and foot movements and self-manipulation and lower rates of head nodding and less facial pleasantness were associated with a more dominant or higher status position relative to the addressee.

Finally, the referential significance of speech activity (e.g., volume or intonation) and facial activity seems to be the degree of involvement with or responsiveness to the addressee. In
2. Facial Activity (0.49): total number of facial expressions including positive and negative ones. Thus, any movements of the facial muscles to a non-neutral expression such as raising of eyebrows in surprise are scored. Continuous expressions are scored once every $5 \mathrm{sec}$.

\section{VERBALIZATION}

1. Communication Length (0.91): measured in terms of number of words, or slightly less satisfactorily in terms of duration which is considerably easier to score.

2. Speech Rate (0.77): measured in terms of words per unit time (e.g. minutes).

3. Halting Quality of Speech (0.70): This is the variability of speech rate and can be computed on the basis of speech rate figures obtained for 15-sec intervals of speech. Alternately, it can be estimated on a 5-point scale by listening to an audio-recording of a communication. Such judgments can be anchored by assigning a score of 0 to "radio announcer" quality speech and a score of 4 to stammering speech. Judges can initially be provided with such examples. The inter-0 reliability given is for the latter case.

4. Speech Error Rate (0.78): based on number of speech disruptions per unit time. Mahl's (1959, Table 1) criteria for "sentence change," "repetition," "stutter," "sentence incompletion," "tongue slips," and "intruding incoherent sounds" provide a reliable basis for scoring speech disruptions.

5. Speech Volume (0.88): obtained, for instance, from the calibrated scale of an audio-recorder or, alternately, simply estimated on a 5-point scale. The inter-0 reliability given is for the batter case where anchor stimuli can again be initially provided to the judges.

6. Intonation (0.44): measured in terms of variations in pitch using a spectrum analyzer such as that described by Starkweather (1964). Alternately, it may be estimated on a 5-point scale with the reported reliability. The latter judgments can be anchored by assigning a score of 0 to "flat or affectless" intonation associated with reading, and a score of 4 to "radio announcer" intonation or that associated with angry speech. These could be initially provided to judges as examples.

general, investigations involving the semantic differential technique (Osgood, Suci, \& Tannenbaum, 1957) have indicated a low positive correlation between activity and evaluation. For example, Bentler (1967) found a 0.33 correlation between activity and evaluation scores assigned to various adjectives. This indicates that a relatively high level of activity (or its referent, responsiveness to the addressee) is more likely to be associated with the communication of positive than of negative feelings. Indeed, Mehrabian and Williams (in press) found that facial activity, speech volume, and intonation were all correlates of intended and perceived persuasiveness and exhibited similar relationships to the latter variables when compared to other positive attitude-communicating cues.

To summarize, our measurement of those nonverbal behaviors that relate to the three referents, evaluation, potency, and responsiveness, includes the following relationships: (1) More positive evaluation or liking is communicated by the more immediate cues of Table 1, and the following cues of Table 2: higher rates of gesticulation, positive head nods, and positive facial expressions; longer communications, higher speech rates, lower rates of speech disturbance, and less halting quality of speech. (2) Potency, social status, or dominance is communicated by the more relaxed cues of Table 1 , and the following cues of Table 2: higher rates of rocking and gesticulation and lower rates of trunk swivel; higher rates of leg and foot movement and 
self-manipulation, lower rates of head nodding, less facial pleasantness, more speech volume, longer communications, and higher speech rates. (3) Finally, responsiveness to the addressee is communicated by the activity cues of Table 2, which are higher rates of facial activity and more speech volume and intonation.

In closing, it should be noted that the measures discussed are primarily based on experiments with North American communicators. Although the findings relating to these measures can be tentatively generalized to communicators in Western cultures, the nonverbal communication codes in these and particularly the less similar (e.g., Oriental) cultures can differ from the North American codes in important respects. For instance, in cultures where status differences are salient cues in social interaction, well defined movements (e.g., bowing) may be more important than postural relaxation in communicating status differences.

\section{REFERENCES}

BENTLER, P. M. Semantic space is (approximately) bipolar. Journal of Psychology, 1969, 71, 33-40.

BIRDWHISTELL, R. L. Introduction to kinetics. Louisville: University of Kentucky Press, 1952.

DAVITZ, J. R. (Ed.). The communication of emotional meaning. New York: McGraw-Hill, 1964.

IKKMAN, P., \& FRIESEN, W. V. Origin, usage, and coding: The basis for five categories of nonverbal behavior. Paper given at the symposium: "Communication theory and linguistic models in the social sciences," at the Center for Social Research at the Torqcuato Di Tella Institute, Buenos Aires, Argentina, October 1967.

HALL, E. T. A system for the notation of proxemic behavior. American Anthropologist, 1963, 65, 1003-1026.

HALL, E. T. The hidden dimension. Garden City, N.Y.: Doubleday, 1966.

KASL, S. V., \& MAHL, G. F. The rclationship of disturbances and hesitations in spontaneous speech to anxiety. Journal of Personality \& Social Psychology, 1965, 1, 425-433.

KRASNER, $L$. Studies of the conditioning of verbal behavior. Psychological Bulletin, 1958, 55, 148-170.

MAHL, G. F. Measuring the patient's anxiety during interviews from "expressive" aspects of his speech. Transactions of the New York Academy of Sciences, 1959, 21, 249-257.

MAHL, G. F., \& SCHULZE, G. Psychological research in the extralinguistic area. In T. A. Sebeok, A. S. Hayes, and M. C. Bateson (Eds.), Approaches to semiotics. The Hague: Mouton, 1964. Pp. 51-124.

MATARAZZO, J. D., WIENS, A. N., \& SASLOW, G. Studies in interviewer speech behavior. In L. Krasner and U. P. Ullman (Eds.), Research in behavior modification. New York: Holt, Rinehart \& Winston, 1965. Pp. 179-210.

MEHRABIAN, A. Communication length as an index of communicator attitude. Psychological Reports, 1965, 17, 519-522.
MEHRABIAN, A. Orientation behaviors and nonverbal attitude communication. Journal of Communication, 1967, 17, 324-332.

MEHRABIAN, A. Inference of attitudes from the posture, orientation, and distance of a communicator. Journal of Consulting \& Clinical Psychology, 1968a, 32, 296-308.

MEHRABIAN, A. Relationship of attitude to seated posture, orientation, and distance. Journal of Personality \& Social Psychology, 1968b, 10, 26-30.

MEHRABIAN, A. Significance of posture and position in the communication of attitude and status relationships. Psychological Bulletin, 1969, 71, 359-372.

MEHRABIAN, A., \& FRIAR, J. T. Encoding of attitude by a seated communicator via posture and position cues. Journal of Consulting \& Clinical Psychology, in press.

MEHR ABIAN, A., \& WILLIAMS, M. Nonverbal concomitants of intended and perceived persuasiveness. Journal of Personality \& Social Psychology, in press.

OSGOOD, C. E. Dimensionality of the semantic space for communication via facial expressions. Scandinavian Journal of Psychology, 1966, 7 1-30.

OSGOOD, C. E., SUCI, G. J., \& TANNENBAUM, P. H. The measurement of meaning. Urbana: University of Illinois Press, 1957.

ROSENFELD, H. M. Approval-seeking and approval-inducing functions of verbal and nonverbal responses in dyad. Journal of Personality \& Social Psychology, 1966a, 4, 597-605.

ROSENFELD, H. M. Instrumental affiliative functions of facial and gestural expressions. Journal of Personality \& Social Psychology, 1966b, 4, 65-72.

SCHLOSBERG, H. Three dimensions of emotion. Psychological Review, $1954,61,81-88$.

SOMMER, R. Small group ecology. Psychological Bulletin, 1967, 67, 145-151.

STARKWEATHER, J. A. Variations in vocal behavior. In D. M. Rioch (Ed.), Disorders of communication. Proceedings of ARNMD, Vol. 42. Baltimore: Williams \& Wilkins, 1964.

TOMKINS, S. S., \& MoCarter, R. What and where are the primary affects? Some evidence for a theory. Perceptual \& Motor Skills, 1964, 18, 119-158.

WIENER, M., \& MEHRABIAN, A. Language within language: Immediacy, a channel in verbal communication. New York: Appleton-CenturyCrofts, 1968.

WILLIAMS, F., \& SUNDENE, B. Dimensions of recognition: Visual vs vocal expression of emotion. Audio Visual Communications Review, $1965,13,44-52$.

WOODWORTH, R. S., \& SCHLOSBERG, H. Experimental psychology. New York: Holt, 1954.

NOTE

1. This study was supported by United States Public Health Service Grant MH 13509. 